\title{
Arylsulfatase A: An Important Metabolic Factor in Pathophysiology of Different Diseases
}

\author{
Kovacs Zsolt*, Tripon Robert, Nemes Nagy Eniko, Balogh Samarghitan Victor, Tilinca Mariana, Martha \\ Orsolya, Fazakas Zita
}

Department of Biochemistry, University of Medicine and Pharmacy Tîrgu Mureș, Romania

\begin{abstract}
Arylsulfatase A (ARSA) is a lysosomal enzyme that plays an important role in catalysis of degradation of cerebrosidesulphate. The deficiency of this lysosomal enzyme causes an autosomal recessive disorder, called metachromatic leucodystrophy. However, a low ARSA activity can be observed in clinically healthy people, called ARSA pseudodeficiency. In our study we investigated the possible linkage between ARSA activity and sulfatide deficiency causing characteristic aspects of degenerative diseases, such as end stage kidney disease, type 2 Diabetes mellitus, Parkinson syndrome, prostate cancer and HIV (Human Immunodeficiency Virus) infection. We used a spectrophotometric method to determine the activity of ARSA. This method of enzyme dosage is based on a 4 hour long hydrolysis of the ARSA enzyme on 4-nitrocatechol sulfate (p-NCS) substrate. The unit of this measurement is $\mathrm{nmol} / \mathrm{ml} / 4 \mathrm{~h}$. Our findings show significant values in type 2 diabetes, Parkinson syndrome and chronic kidney disease. The importance of sulfatide in these diseases is well-known, thus presumably the variation of the ARSA's activity might play an important role in the pathophysiology of these diseases, involving a vicious cycle between sulfatide degradation andthese diseases.
\end{abstract}

Keywords: arylsulfatase A, sulfatide, chronic kidney disease, type 2 diabetes, Parkinson syndrome, prostate cancer, HIV infection.

Received: 09 december 2014 / Accepted: 18 July 2015

\section{Introduction}

Arylsulfatase A is a lysosomal enzyme that catalyzes cerebrosidesulphate degradation [1]. The synthetisation of galactosylceramide $(\mathrm{GalC})$ takes place in the endoplasmic reticulum in presence of the UDP-galactose (Uridinediphosphategalactose), which catalyzes the transfer of galactose to ceramide molecule. GalC is converted to sulfatide in the Golgi apparatus, by transferring a sulfate group from PAPS (3'-Phosphoadenosine-5'-phosphosulfate) to the galactose residue, which is catalyzed by the Gal3st1 (Galactosylceramide sulfotransferase) (Figure 1). The degradation of sulfatide starts with the hydrolysis of the sulfate residue by ASA [2]. The resulting molecules are re-used as precursors for sulfatide biosynthesis. When ARSA does not hydrolyze properly, this recycling pathway breaks down.

In our study we investigated the possible linkage between ARSA activity and sulfatide deficiency causing characteristic aspects of degenerative diseases, such as end stage kidney disease, type 2 Diabetes mellitus, Parkinson syndrome, prostate cancer and HIV infection.

\section{Methods}

We included 174 patients in our study, divided into six groups according to the examined pathologies as follows: 58 patients presenting end stage kidney failure, 24 patients with type 2 diabetes, 19 patients diagnosed with prostate cancer (histologically confirmed), 14 patients presenting Parkinson syndrome, 27 HIV positive patients and 32 pa-

* Correspondence to: Zsolt Kovacs

E-mail: kovacska_zsoltkovacs@yahoo.com tients representing the healthy control group. Out of the patients with chronic kidney disease, a separate group of 12 individuals were identified with positive Minimental state exam test (MMSE) and the Clock drawing test for dementia.

\section{Lab procedure and Measurements}

Peripheral blood samples were collected from the patients in sodium-heparin tubes. The serum was isolated by spinning the blood tubes in a centrifuge at $2500 \mathrm{rpm}$ for 10 minutes at room temperature, as described in the CMCI (Center of molecular and cellular intervention) Utrecht protocol. Until further processing of the serum, the samples were stored at $-20^{\circ} \mathrm{C}$.

The method of enzyme dosage is based on a 4 hour long hydrolysis of the arylsulfatase A on the 4-nitrocatechol sul-

\section{Ceramide

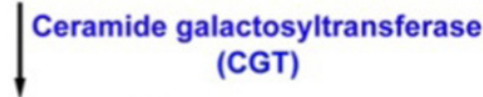

$\begin{gathered}\text { Arylsulfatase A } \\ \text { (ASA) } \\ \text { Saposin B (SapB) }\end{gathered}|| \begin{array}{ll}\text { Cerebroside sulfotransferase } \\ \text { (CST) }\end{array}$
PAPS

3-O-Sulfogalactosylceramide (Sulfatide)

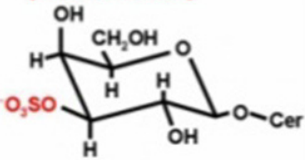

Fig, 1.Role of ARSA in biosynthesis of sulfatide [3] 
fate (p-NCS) substrate. We obtained the reagent in several steps. First, we added $6.3 \mathrm{ml}$ of acetic acid to $100 \mathrm{ml}$ of distilled water resulting the solution $\mathrm{A}$. Then, $6.8 \mathrm{~g}$ sodium acetate was mixed with $50 \mathrm{ml}$ of distilled water resulting the solution B. Three $\mathrm{ml}$ of solution A mixed with $7 \mathrm{ml}$ of the solution $\mathrm{B}$ gave us the solution $\mathrm{C}$, with a $\mathrm{pH}$ of 5 , optimal for the ARSA's activation. The last step was to add $2.2 \mathrm{mg}$ inorganic pyrophosphate, $62 \mathrm{mg}$ p-NCS and $1 \mathrm{~g}$ potassium chloride to the solution $\mathrm{C}$, obtaining the needed reagent with the substrate.

Once this step was completed, we defrosted the serum samples and we set up the probes and controls for the spectrophotometry. The probe was $0.3 \mathrm{ml}$ of the reagent mixed with $0.3 \mathrm{ml}$ of the serum of each patients individually, incubated for 4 hours at $37^{\circ} \mathrm{C}$ using Termostat Bath FALC. The control for this reaction was the incubation of the two compounds separately following the same conditions, and mixing them right after the incubation. At the end of the incubation we added $0.6 \mathrm{ml}$ of sodium hydroxide $1.25 \mathrm{~N}$ (Normal) to both probe and control samples in order to stop the ARSA's hydrolysation on the substrate.

The extinction of the probes were measured in relation to the controls for each patient. For this step, we used the $T$ $60 \mathrm{UV}-\mathrm{Visible}$ spectrophotometer at $515 \mathrm{~nm}$ wavelength. The unit of measurement used for the ARSA substrate concentration $\mathrm{p}-\mathrm{NCS}$ was $\mathrm{nmol} / \mathrm{ml} / 4 \mathrm{~h}$.

This spectrophotometric method was choosed in accordance with publised data, which indicate that this is the most accessible and cost-effective method available

Statistical analyses were performed including Grubbs test for outliers, normality test and t-test using Graph$\mathrm{Pad}$ and descriptive analysis was made in Microsoft Office 2010 [4].

\section{Results and discussions}

Our findings suggest a wide enzyme activity range, which fits the Gaussian distribution (Table 1, Figure 2) and the absence of statistically significant difference between female and male patients enzyme's activity $(\mathrm{p}=0.29)$.

Published data indicate a wide normal ARSA activity, similar to our findings [5]. The case of ARSA pseudodeficiency has been described in the literature, representing clinically healthy people who present reduced enzyme activity [6].

\section{ARSA activity in type 2 Diabetes mellitus}

The low serum concentration of sulfatide in patients diagnosed with type 2 Diabetes mellitus has been described in the literature [7]. A statistically significant difference was described as compared to the control group (Table 1), indicating a reduced ARSA activity in these patients. These findings suggest a vicious cycle where presence of diabetes lowers ARSA activity, leading to a decrease in the sulfatide biosynthesis and the absence of sulfatide worsening the diabetes.

\section{ARSA activity in Parkinson syndrome}

Our findings denotes a lower enzymatic activity in case of patients with Parkinson syndrome $(\mathrm{p}=0.002)$. Similar findings were described in the literature [8] indicating that ARSA deficit may be a metabolic factor associated with the clinical chaacteristics of this disease.

\section{ARSA activity in chronic kidney disease}

Low serum sulfatide level was found in patients with chronic kidney disease, receiving hemodialysis over a long period [9]. An animal model study, modeling an acute kidney failure through protein overload nephropathy, found that serum sulfatide level is dependent on the hepatic se-

Table 1. ARSA activity: mean, standard deviation, minimum value, maximum value and $\mathrm{p}$ coefficient related to control group

\begin{tabular}{lccccc}
\hline & Mean & SD & Min & Max & $p$ \\
\hline Control & 2.46 & 1.76 & 0.4 & 5.3 & \\
Diabetes & 5.64 & 3.93 & 1.9 & 16 & 0.01 \\
Parkinson & 5.87 & 3.01 & 2 & 12 & 0.002 \\
Chronic kidney & 4.86 & 2.44 & 1.78 & 12.5 & 0.025 \\
disease & & & & & \\
HIV inf. & 4.5 & 2.88 & 0.5 & 11 & 0.091 \\
Prostate cancer & 5.1 & 2.71 & 0.7 & 8.9 & 0.01 \\
\hline
\end{tabular}
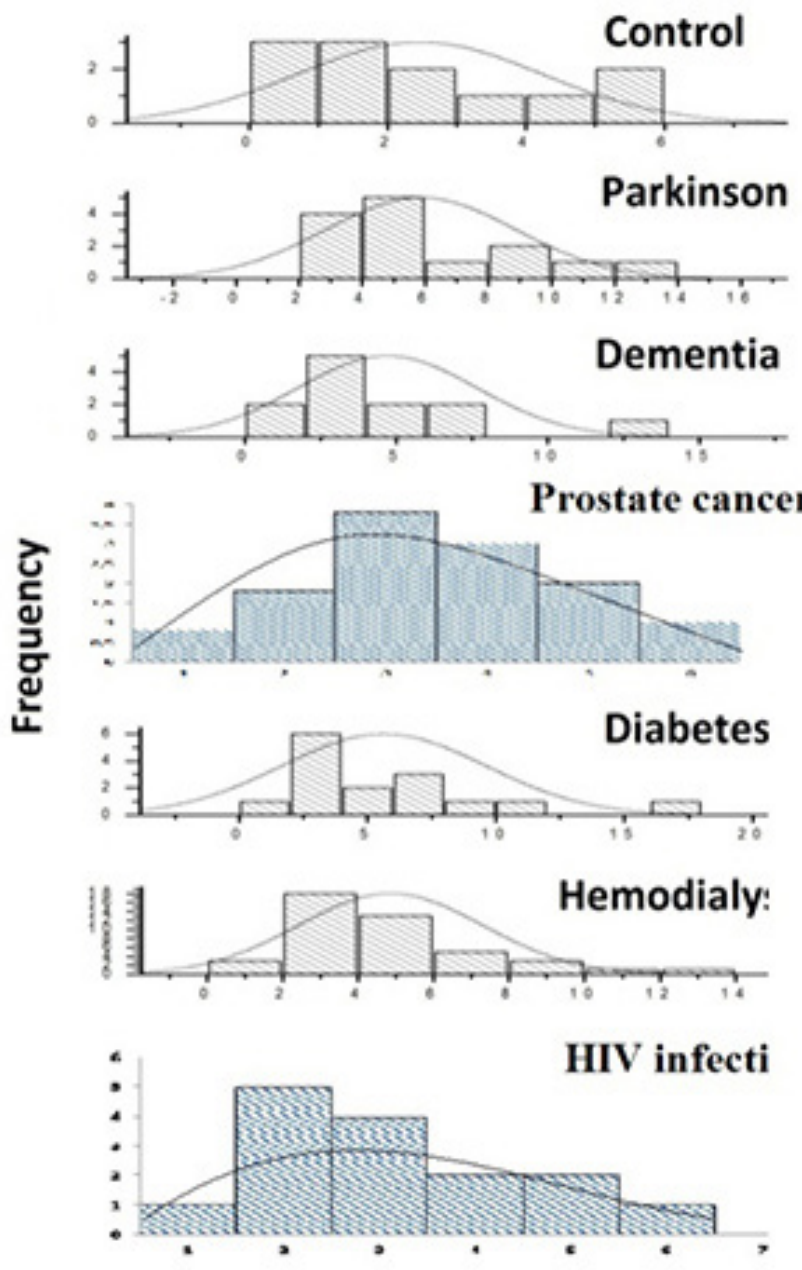

Fig. 2. Gaussian distribution of ARSA activity in the studied groups 
cretory ability of sulfatide. Cerebroside sulfotransferase regulates this process with hepatic gene expression, which in this case is down-regulated [10]. Our findings show a decay of ARSA activity $(\mathrm{p}=0.025)$ that correlates with the years of dialysis treatment $(\mathrm{p}=0.001)$.

\section{ARSA activity in HIV infected patients}

An association between low sulfatide level and Alzheimer's disease has been described in the literature $[11,12]$. However, anti-sulfatide antibodies have been identified in HIV positive patients with neurological symptoms [13]. We did not find any significant difference between HIV infected patients and our control group $(\mathrm{p}=0.091)$, however there was a statistically significant difference between HIV infected patients with and without neurological symptoms $(\mathrm{p}=0.001)$.

\section{ARSA activity in prostate cancer}

In case of prostate cancer patients, ARSA shows a wide range of enzyme activity which follows Gaussian distribution.

The scientific literature describes a wide normal ARSA activity range, similar to our findings. It was shown that these variations are caused by allelic mutations, but have no consequences on human health. We call pseudodeficiency the case when clinically healthy individuals have reduced enzyme activity. It was suggested that even a low activity $(<10 \%$ of the mean normal) is sufficient for in vivo hydrolysis and hormone activation, playing a role in cancer development. The scientific opinion is that the enzymes involved in hormone synthesis are cancer therapy targets and potent STS (steroid sulfatase) inhibitors should be used for therapeutic purpose in hormone-dependent cancers and other non-oncological conditions [14,15].

\section{Conclusions}

These findings suggest a new hypothesis in which a vicious cycle is enclosed between ARSA, sulfatide and degenerative diseases. ARSA may be an important metabolic co-factor of clinically different diseases, but with common molecular physiopathology. Our findings should increase clinician's attention towards possible complications in these patients as a consequence of secondary sulfatide disorders.

\section{References}

1. Barth M L, Ward C, Harris A, Saad A, Fensom A.- Frequency of arylsulphatase A pseudodeficiency associated mutations in a healthy population. J Med Genet, 1994;31:667-671.

2. Ramakrishnan $\mathrm{H}$, Hedayati KK. Increasing sulfatide synthesis in myelin forming cells of arylsulfatase A deficient mice causes demyelinisation and neurological symptoms reminiscent of human metachromatic leucodystophy. J Neurosci, 2007;27:9482-9490.

3. Takahasi T, Murakami K, et al. Sulfatide is required for efficient replication of Influenza A virus. J Virol, 2008;82:5940-5950.

4. Fazakas Z, Tilinca M, Nemes-Nagy E, et al. Arylsulphatase A - A possible important determinant in the pathophysiology of metabolic and neuropsychiatric diseases. Annals of RSBC, 2013;1:80-84.

5. Thomas GH. Commentary "Pseudodeficiencies" od lysosomal hydrolases. Am J Hum Genet, 1994; 54:934-940.

6. Wenger D, Louie E. Pseudodeficiencies of arylsulfatase A and galactocerebrosidase activities. Dev Neurosci, 1991;13:216-221.

7. Buschard K, Blomqvist V, Osterbye T, Fredman P. Involvement of sulfatide in beta cells and type 1 and type 2 diabetes. Diabetologia, 2005;48:19571962.

8. Martinelli P, Ippoliti M, Montanari M, et al. Arylsulphatase A (ASA) activity in parkinsonism and symptomatic essential tremor. ActaNeurolScand, 1994;89:171-174.

9. Hu R, Li G, Kamijo Y. Serum sulfatides as a novel biomarker for cardiovascular disease in patients with end-stage renal failure. Glicoconj J, 2007;24:565-571.

10. Zhang $X$, Nakajima T, Kamijo $Y$, et al. Acute kidney injury induced by protein overload nephropathy down-regulates gene expression of hepatic cerebroside sulfotransferase in mice, resulting in reduction of liver and serum sulfatides, Biochem Biophys Res Commun, 2009;390:13821388.

11. Han X, Holtzman D, Mckeel DW. Substantial sulfatide deficiency and ceramide elevation in very early Alzheimer's disease: potential role in disease pathogenesis, J Neurochem, 2002;82:809-818.

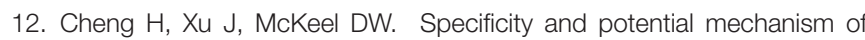
sulfatide deficiency in Alzheimer's disease: an electrospray ionization mass spectrometric study, Cel Mol Biol; 2003;49:809-818.

13. Petratos S, Turnbull VJ, Papadopoulos R, Ayers M, Gonzales MF Antibodies against peripheral myelin glycolipids in people with HIV infection. Immun Cell Biol, 1998;76:535-541.

14. Purohit A, Foster PA. Steroid sulfatase inhibitors for estrogen- and androgen-dependent cancers. J Endocrinol. 2012;212:99-110.

15. Bhattacharyya S, Tobacman JK. Steroid sulfatase, arylsulfatases A and $B$, galactose-6-sulfatase, and iduronate sulfatase in mammary cells and effects of sulfated and non-sulfated estrogens on sulfatase activity. J Steroid Biochem Mol Biol. 2007;103:20-34. 